\title{
ELECTRONIC PAPER
}

\section{Intervention strategies to reduce musculoskeletal injuries associated with handling patients: a systematic review}

\section{S Hignett}

Occup Environ Med 2003;60:e6(http://www.occenvmed.com/cgi/content/full/60/9/e6)

Correspondence to: Dr S Hignett, Lecturer in Ergonomics, Dept of Human Sciences,

Loughborough University, Leicestershire LE 11 3TU, UK;

S.M.Hignett@lboro.ac.uk

Accepted

14 February 2003

\begin{abstract}
Aims: To report, analyse, and discuss the results of a systematic review looking at intervention strategies to reduce the risk factors associated with patient handling activities.

Methods: A search strategy was devised to seek out research between 1960 and 2001. Inclusion/ exclusion criteria limited the entry of papers into the review process. A checklist was selected and modified to include a wide range of study designs. Inter-rater reliability was established between six reviewers before the main review process commenced. Each paper was read by two reviewers and given a quality rating score, with any conflicts being resolved by a third reviewer. Papers were grouped by category: multifactor, single factor, and technique training based interventions.

Results: A total of 2796 papers were found, of which 880 were appraised. Sixty three papers relating to interventions are reported in this paper. The results are reported as summary statements with the associated evidence level (strong, moderate, limited, or poor).

Conclusion: There is strong evidence that interventions predominantly based on technique training have no impact on working practices or injury rates. Multifactor interventions, based on a risk assessment programme, are most likely to be successful in reducing risk factors related to patient handling activities. The seven most commonly used strategies are identified and it is suggested that these could be used to form the basis of a generic intervention programme, with additional local priorities identified through the risk assessment process. Health care providers should review their policies and procedures in light of these findings.
\end{abstract}

$\mathrm{P}$ atient handling activities have long been acknowledged as being a major contributor to the high incidence of musculoskeletal injury, in particular low back pain, in health care staff. ${ }^{1}$ A range of intervention strategies have been used over the years to try and reduce this problem, ${ }^{2}$ and professional bodies continue to produce guidance on patient handling. ${ }^{3-7}$ These guidance publications have tended to promote technique training as the main factor of the intervention programme, although more recently risk management programmes are evident.

This paper summarises a section of the results of a systematic review on patient handling tasks, equipment, and interventions that sought to develop a foundation from which evidence based guidelines could be developed. The following research questions were addressed:

(1) Can research be found on patient handling tasks, equipment, and interventions?

(2) What are the results from the research?

(3) How do these results compare with the current guidance available?

\section{Main messages}

- An international systematic review found 63 papers relating to intervention strategies to reduce the risk of musculoskeletal injuries associated with patient handling.

- There is strong evidence that interventions for patient handling based on technique training have no impact on working practices or injury rates.

- Multifactor interventions, based on a risk assessment programme, are most likely to be successful in reducing risk factors associated with patient handling activities.

- Seven strategies are suggested for inclusion in a generic intervention programme.
The review produced evidence statements in a similar process to that undertaken by the Faculty of Occupational Medicine. ${ }^{8}$ The revision and development of new guidelines is currently being considered by the Royal College of Nursing Advisory Panel for Back Pain.

This paper summarises and analyses the results relating to intervention strategies.

\section{METHODS}

The systematic review process is described in detail elsewhere. ${ }^{10}$ A search strategy was developed with assistance from the Trent Institute for Health Services Research, University of Nottingham and the NHS Centre for Reviews and Dissemination of Information, University of York. This included the following main search terms (in appropriate combinations): patient, manual, handl*, lift, mov*, transfer, carr*, toilet, hospital bed, bath, nurs*, (body region) injuries, ergonomic*, equipment and supplies etc. The search string was run on: Medline (1960-2001), AMED, Psychinfo, Ergonomics Abstracts, EMBASE, CINAHL, British Nursing Index, and Best Evidence. Additional references were sought by hand searching journals and exploding the reference list of identified papers, contacting expert informants (dissertations and theses), and searching personal collections.

The review intentionally included both quantitative and qualitative data sources. All languages were included in the

\section{Policy implications}

- Health care providers should review their policies and pro cedures in light of this systematic review.

- Interventions predominantly based on technique training are unlikely to be successful in reducing musculoskeletal injuries, so an alternative strategy should be considered. 
Table 1 Evidence levels

++++ Strong evidence: provided by multiple (three or more), high quality (QR $\geqslant 75 \%)$ studies

$+++\quad$ Moderate evidence: provided by generally consistent findings in fewer (two or more), smaller or lower quality (QR $=50-74 \%)$ studies

$++\quad$ Limited or contradictory evidence: provided by one study (QR $\geqslant 50 \%)$, or findings in multiple (two or more) lower quality (QR $=25-49 \%)$ studies

$+\quad$ Poor or no evidence: no studies or low quality score (QR $\leqslant 24 \%$ )

search which resulted in 30 papers being translated from Chinese, Danish, Dutch, French, German, Italian, Japanese, Norwegian, Portuguese, Slovakian, and Spanish.

The data extraction/critical appraisal tool used was developed by Downs and Black $^{10}$ for randomised and nonrandomised studies of health care interventions. This has four sections:

(1) General structure of paper to include 10 questions about the aims, sampling, method (description of intervention), outcome measures, confounders, findings, analysis, and discussion of adverse events.

(2) External validity is appraised using three questions about the representativeness of the sample and context of the study.

(3) Internal validity (bias) includes seven questions to look at blinding of subjects/data collectors, compliance with the intervention, choice of outcome measures, and statistical tests.

(4) Internal validity (confounding, selection bias) has six questions looking at the sampling strategy with respect to diversity within the recruitment population and chronology of the study. This section also addresses issues about the allocation to control/experimental groups and subject follow up.

This appraisal tool was further extended and modified to include observational studies without an intervention (cohort studies, case-control studies, cross sectional studies, surveys, and case series) and an additional section for qualitative studies. Before the review process started an inter-reliability study was carried out with the six reviewers. This resulted in an overall intra-class correlation (pairwise) of $0.95 .{ }^{11}$

Each paper was sent to two reviewers following a screening process to ensure that reviewers did not receive their own publications. If the difference in the quality rating scores exceeded an established limit the paper was sent to a third reviewer for conflict resolution. Owing to the heterogeneity of the study types, interventions, settings, participants, outcome measures, and comparison groups a quantitative analysis (meta-analysis) was not appropriate.

The data were synthesised in two stages. The first involved grouping papers into tasks, equipment, and interventions, with some papers being allocated to more than one section. The second stage involved combining the papers to produce summary statements and then allocating evidence levels. The evidence levels (table 1) were developed using concepts from Bernard $^{12}$ and the Faculty of Occupational Medicine. ${ }^{8}$

A total of 2796 papers were located. These were then checked to eliminate duplications (from the different search strategies) and papers which were inappropriate to the research topic based on their title (for example, working postures of dentists). The remaining 880 papers were included, and sent to the project team for review. Subsequent eliminations were based on the following inclusion/exclusion criteria, whereby a paper or document was:

(1) Included if it described a named task, piece(s) of equipment, or intervention relating directly to patient handling.

(2) Included as a professional opinion if it:

- had references

- critically appraised the literature

- provided a new interpretation of the literature.
(3) Excluded if it was related to epidemiology of musculoskeletal disorders (usually low back pain) and did not meet criterion (1) for the study.

(4) Excluded if it was not the primary source of a study. The primary source was sought and included.

(5) Excluded if it was a legal case law report.

A total of 225 papers were included in the full project review, ${ }^{9}$ with the 63 papers relating to intervention strategies being reported in this paper.

\section{RESULTS}

The findings of the 63 papers (table 2 ) have been grouped into three categories for the summary evidence statements.

(1) Multifactor interventions.

(2) Single factor interventions.

(3) Technique training based interventions.

Any conflicting and negative evidence has been included in the evidence statement for categories ( 1 ) and (2). Category (3) is subdivided into three further subgroupings to present negative, mixed, and positive evidence.

\section{Multifactor interventions}

A decision was taken to present the data in this category as two groups to look at the role of risk assessment as part of an intervention strategy. This will be reviewed in the discussion. +++ The evidence statement that multifactor interventions based on risk assessment are successful is supported at a moderate level by 10 studies, ${ }^{13-22}$ and at a limited level with an additional four studies. ${ }^{23-26}$

+++ The evidence statement that multifactor interventions (not based on risk assessment) can show improvements is supported with moderate evidence from four studies. ${ }^{27-30}$ Additional limited evidence is available from five studies. ${ }^{31-35}$ However, there is also contradictory evidence from one high quality study ${ }^{36}$ which found no improvement using a multifactor intervention.

\section{Single factor interventions}

+++ The evidence statement that single factor interventions based on the provision of equipment can be effective is supported with moderate evidence from two studies. ${ }^{37} 38$

+++ The evidence statement that interventions using the lifting team approach can be effective is supported with moderate evidence from three studies. ${ }^{39-41}$ Additional support is available at the limited evidence level from two studies. ${ }^{42} 43$

\section{Interventions predominantly based on technique training}

++++ The evidence statement that interventions based predominantly on technique training have no impact on working practices or injury rates is supported with strong evidence from four studies. ${ }^{44-47}$ Eight additional studies give a moderate level of support. ${ }^{48-55}$ There are also five studies at the limited evidence level supporting this statement. ${ }^{56-60}$

+++ The evidence statement that interventions based on technique training can have mixed (positive and negative) short term 
Table 2 Summary of interventions and critical appraisal (QR) scores

\begin{tabular}{|c|c|c|c|c|}
\hline Author & Intervention subjects ( $\mathrm{n}$ ) & Outcome measures & Results & QR \\
\hline Addington $(1994)^{63}$ USA & $\begin{array}{l}5,22 \\
\text { Operating room staff ( } n=?)\end{array}$ & $\begin{array}{l}\text { No. of reported back injuries } \\
\text { Restricted working days }\end{array}$ & $\begin{array}{l}\text { No decrease in injuries } \\
\text { Reduction in restricted days }\end{array}$ & $37 \%$ \\
\hline Aird $(1988)^{31}$ Canada & $\begin{array}{l}\text { Hospital: } 2,5,9,12,18,20,21 \\
\text { Home for the Aged: } 1,3,5,13 \\
(n=?)\end{array}$ & $\begin{array}{l}\text { Lost time injury claims } \\
\text { (Workers Compensation Board) }\end{array}$ & $\begin{array}{l}\text { Hospital: Back injuries reduced by (a) number }(8.4 \%) \text {, (b) frequency } \\
\text { (18.8\%) } \\
\text { Home for the Aged: No back injuries in } 12 \text { months following } \\
\text { intervention }\end{array}$ & $44 \%$ \\
\hline Alavosius and Sulzer-Azaroff $(1986)^{71}$ USA & $\begin{array}{l}5,8 \\
\text { Direct care staff }(n=6)\end{array}$ & No. of safe transfers & Reduction in no. of unsafe transfers from 13 to 4 & $39 \%$ \\
\hline Alexander $(1996)^{13}$ UK & $\begin{array}{l}1,2,6,11,13,16 \\
\text { Community nurses }(n=42)\end{array}$ & $\begin{array}{l}\text { Relationship between implementation of } \\
\text { recommendations and level of sickness absence }\end{array}$ & $\begin{array}{l}\text { Significant relationship between implementation of recommendations } \\
\text { and reduction in sickness absence }\end{array}$ & $50 \%$ \\
\hline Best $(1997)^{67}$ Australia & $\begin{array}{l}5 \\
\text { Nursing Home }(n=55)\end{array}$ & $\begin{array}{l}\text { Postural analysis } \\
\text { Back pain (severity and frequency) } \\
\text { Rated Perceived Exertion (RPE) }\end{array}$ & All reduced but not significantly & $70 \%$ \\
\hline Billin $(1998)^{48}$ UK & $\begin{array}{l}2,5 \\
\text { Nurses, Occupational Therapists, } \\
\text { Physiotherapists }(n=?)\end{array}$ & Moving and handling injuries & Increase in injuries over 5 year period & $54 \%$ \\
\hline Caska et al $(1998)^{39}$ USA & Medical ward $(n=4)$ & $\begin{array}{l}\text { Effectiveness of liffing team } \\
\text { Injury rate }\end{array}$ & $\begin{array}{l}\text { Team completed } 94 \% \text { of scheduled and paged lifts } \\
\text { No musculoskeletal discomfort reported by the team }\end{array}$ & $69 \%$ \\
\hline Charney $(1997)^{40}$ USA & $\begin{array}{l}17 \\
\text { Hospital staff ( } n=10 \text { units) }\end{array}$ & $\begin{array}{l}\text { Incident rates } \\
\text { Lost working time }\end{array}$ & Reduction in incident rates (by 63\%) and lost work days (by $90 \%$ ) & $72 \%$ \\
\hline Charney et al $(1993)^{41}$ USA & Orderlies $(n=2)$ & $\begin{array}{l}\text { Accident rate } \\
\text { Sickness absence }\end{array}$ & $\begin{array}{l}\text { Year } 2 \text { data: No injuries or sick leave for liffing team } \\
\text { Nursing sick leave was reduced }\end{array}$ & $61 \%$ \\
\hline Charney et al $(1991)^{42}$ USA & Orderlies $(n=2)$ & Accident rate & $\begin{array}{l}\text { Year } 1 \text { data: Reduced from } 39 \text { to } 2.4 \text { cases }(62 \%) \text { with a projected } \\
\text { saving of } \$ 65,000 \text { per annum }\end{array}$ & $37 \%$ \\
\hline Collins $(1990)^{14}$ Australia & $\begin{array}{l}1,5,12,13,14 \\
\text { Nurses (n=?) }\end{array}$ & Sickness absence & Reduced from 17 to 11 working days per claim & $52 \%$ \\
\hline Daws $(1981)^{64}$ UK & $\begin{array}{l}5 \\
\text { Nurses }\end{array}(n=2000)$ & Injury rate & No change & $31 \%$ \\
\hline Daynard et al $(2001)^{27}$ Canada & $\begin{array}{l}2,5 \\
\text { Hospital staff }(\mathrm{n}=36)\end{array}$ & $\begin{array}{l}\text { Compliance with intervention } \\
\text { Biomechanical evaluation of spinal loading }\end{array}$ & $\begin{array}{l}\text { Increased compliance } \\
\text { Reduced spinal loading }\end{array}$ & $50 \%$ \\
\hline Dietz and Baumann $(2000)^{56}$ France & $\begin{array}{l}5 \\
\text { Nurses and physiotherapists }(n=103)\end{array}$ & Training impact & $\begin{array}{l}76 \% \text { felt they had not learned the basic positions at the end of the } \\
\text { course }\end{array}$ & $33 \%$ \\
\hline Dixon et al $(1996)^{32}$ UK & $\begin{array}{l}2,5,10 \\
\text { Ward staff ( } n=?)\end{array}$ & Musculoskeletal sickness absence & No episodes of sickness absence after implementation & $20 \%$ \\
\hline Duggan $(1995)^{15}$ Ireland & $\begin{array}{l}1,2,5,6,7 \\
\text { Nurses }(n=24)\end{array}$ & $\begin{array}{l}\text { Postural analysis } \\
\text { RPE }\end{array}$ & Significant reduction in harmful postures and RPE & $74 \%$ \\
\hline Engels et al $(1998)^{65}$ Netherlands & $\begin{array}{l}5,8,10 \\
\text { Nurses }(n=24)\end{array}$ & $\begin{array}{l}\text { Postural load } \\
\text { Ergonomic and biomechanical errors } \\
\text { RPE }\end{array}$ & $\begin{array}{l}\text { Both postural load and errors decreased significantly } \\
\text { RPE increased }\end{array}$ & $44 \%$ \\
\hline Engkvist et al $(2001)^{44}$ Sweden & $\begin{array}{l}2,5 \\
\text { Nursing staff }(n=292)\end{array}$ & $\begin{array}{l}\text { Interaction between risk factors for back injuries and } \\
\text { training }\end{array}$ & No association with decreased risk of injury & $100 \%$ \\
\hline Entwhistle et al $(1996)^{33}$ UK & $\begin{array}{l}2,5,10,13,22 \\
\text { Nurses }(n=900)\end{array}$ & Lost working time & Reduction in certified illness from 35 to 8 episodes per annum & $35 \%$ \\
\hline Evanoff et al $(1999)^{16}$ USA & $\begin{array}{l}\text { 1, 3, } 4,6,7,9,10 \\
\text { Hospital orderlies }(n=67)\end{array}$ & $\begin{array}{l}\text { Reportable injuries (OSHA } 200 \text { log) } \\
\text { Workers compensation insurance records } \\
\text { Self-administered survey }\end{array}$ & $\begin{array}{l}\text { Reduction in injury rate from } 32.5 \text { per } 100 \text { FTE to } 16.3 \text { per } 100 \text { FTE } \\
\text { Relative risk reduced by } 50 \% \\
\text { No significant findings for workers compensation records } \\
\text { Significant reduction in proportion of employees with musculoskeletal } \\
\text { symptoms }\end{array}$ & $58 \%$ \\
\hline Fanello et al $(1999)^{45}$ France & $\begin{array}{l}5 \\
\text { Non-clerical hospital staff }(n=272)\end{array}$ & $\begin{array}{l}\text { Injury rate (musculoskeletal disorders) } \\
\text { Amount of patient handling } \\
\text { Postural analysis }\end{array}$ & No significant difference for all three measures & $80 \%$ \\
\hline Feldstein et al $(1993)^{68}$ USA & $\begin{array}{l}5,18 \\
\text { Nurses, aids and orderlies }(n=55)\end{array}$ & $\begin{array}{l}\text { Back pain } \\
\text { Quality of patient transfers }\end{array}$ & $\begin{array}{l}\text { Reduction (not significant) } \\
19 \% \text { improvement in transfers }\end{array}$ & $68 \%$ \\
\hline Paternoster et al $(1999)^{73}$ Italy & $\begin{array}{l}5,18 \\
\text { Hospital workers }(n=80)\end{array}$ & Postural analysis & Incorrect postures reduced from $68 \%$ to $38 \%$ & $31 \%$ \\
\hline
\end{tabular}

Non-clerical hospital staff $(\mathrm{n}=272)$

5,18
Nurses, aids and orderlies $(n=55)$

Hospital workers $(n=80)$ of patient transfers

Postural analysis 
Table 2 continued

\begin{tabular}{|c|c|c|c|c|}
\hline Author & Intervention subjects (n) & Outcome measures & Results & QR \\
\hline Foster $(1996)^{69} \mathrm{UK}$ & $\begin{array}{l}5 \\
\text { Nurses }(n=100)\end{array}$ & $\begin{array}{l}\text { Change in practice } \\
\text { Use of equipment }\end{array}$ & $\begin{array}{l}74 \% \text { change in practice } \\
77 \% \text { improved use of equipment }\end{array}$ & $57 \%$ \\
\hline Garg and Owen (1992) 17 USA & $\begin{array}{l}1,2,5 \\
\text { Nursing Homes }(n=57)\end{array}$ & Incidence of back injuries & Reduced from 83 to 47 per 200,000 work hours & $63 \%$ \\
\hline Garrett and Perry $(1996)^{66}$ USA & $\begin{array}{l}1,5,10,12,15 \\
\text { Nursing and therapy staff }(\mathrm{n}=700)\end{array}$ & Lost working time cases & Reduced from 42 to 23 per annum & $46 \%$ \\
\hline Goodridge and Laurila $(1997)^{23}$ Canada & $\begin{array}{l}2,13 \\
\text { Nurses }(n=?)\end{array}$ & Injury rate & $\begin{array}{l}\text { Reduction in injury rate from } 6.7 \text { to } 4.1 \text { patient handling injuries per staff } \\
\text { member per month }\end{array}$ & $44 \%$ \\
\hline Gray et al $(1996)^{72}$ Canada & 5 & Knowledge of procedures & Significant improvement & $43 \%$ \\
\hline Griffith and McArthur (1999) $)^{57}$ UK & $\begin{array}{l}5 \\
\text { Health care assistants }(n=502)\end{array}$ & Impact of training using questionnaire & $\begin{array}{l}\text { No acquisition of transferable skills with respect to applying the techniques } \\
\text { in different environments }\end{array}$ & $42 \%$ \\
\hline Harber et al $(1994)^{49}$ USA & $\begin{array}{l}5 \\
\text { Newly qualified nurses }(n=179)\end{array}$ & Association between training and future back pain & No association & $73 \%$ \\
\hline Head and Levick (1996) $)^{24}$ Australia & $\begin{array}{l}1,2,3,5 \\
\text { Nurses and ambulance workers ( } \mathrm{n}=\text { ?) }\end{array}$ & No. of back injury claims & $\begin{array}{l}\text { Reduction in number (by } 23 \% \text { ), lost time (by } 38 \% \text { ) and average cost (by } \\
56 \% \text { ) of back injury claims }\end{array}$ & $28 \%$ \\
\hline Hellsing ef al $(1993)^{61}$ Sweden & $\begin{array}{l}5,18,19 \\
\text { Nursing students }(n=51)\end{array}$ & $\begin{array}{l}\text { Nordic Questionnaire } \\
\text { Observation of standardised work tasks }\end{array}$ & $\begin{array}{l}\text { No short term effects on musculoskeletal problems } \\
\text { Reduction of lifts (and shorter times) in extreme positions }\end{array}$ & $58 \%$ \\
\hline Hignett and Richardson $(1995)^{18} \mathrm{UK}$ & $\begin{array}{l}1,3,5,6,7,9,10 \\
\text { Nurses }(n=26)\end{array}$ & Qualitative & Risk assessment model & $81 \%$ \\
\hline Holliday et al $(1994)^{37}$ Canada & 2 Nursing staff $(n=22)$ & $\begin{array}{l}\text { No. of staff for a task } \\
\text { RPE } \\
\text { Comfort } \\
\text { Time taken }\end{array}$ & $\begin{array}{l}\text { Fewer staff needed and significant reduction in RPE } \\
\text { No change in comfort or time taken }\end{array}$ & $50 \%$ \\
\hline Johnston $(1987)^{58}$ UK & $\begin{array}{l}5 \\
\text { Student nurses }(n=7)\end{array}$ & Application of training principles & $\begin{array}{l}\text { Only } 28 \% \text { of lifts were planned } \\
\text { Assistance was used for } 50 \% \text { of lifts }\end{array}$ & $43 \%$ \\
\hline Kilbom et al $(1985)^{34}$ Sweden & $\begin{array}{l}2,6,7 \\
\text { Home care nurses }(n=12)\end{array}$ & $\begin{array}{l}\text { Vertical force and duration of lift, weight distribution and } \\
\text { no. of steps while carrying }\end{array}$ & $\begin{array}{l}\text { The modern ward showed a reduction in: total weight }(43 \%) \text {; no. of lifts } \\
\text { per hour }(53 \%) \text {; asymmetric lifts }(60 \%) \text {; and no. of steps while carrying } \\
(73 \%) \text {; }\end{array}$ & $27 \%$ \\
\hline Knibbe and Friele $(1999)^{38}$ Netherlands & $\begin{array}{l}2 \\
\text { Home care nurses }(n=378)\end{array}$ & $\begin{array}{l}\text { Prevalence of back pain ( } 12 \text { months) } \\
\text { Lift Counter (self-administered log) }\end{array}$ & $\begin{array}{l}\text { Significant reduction in back pain (from } 74 \text { to } 64 \% \text { ) } \\
\text { Reduction in total no. of transfers from } 35 \text { to } 21 \text { per nurse per week }\end{array}$ & $83 \%$ \\
\hline Lagerström and Hagberg $(1997)^{46}$ Sweden & $\begin{array}{l}2,5,18,19 \\
\text { Nurses }(n=348)\end{array}$ & $\begin{array}{l}\text { Questionnaire on musculoskeletal symptoms, physical } \\
\text { fitness and physical workload }\end{array}$ & $\begin{array}{l}\text { No reduction in neck, shoulder and back symptoms, increase in hip and } \\
\text { upper back problems. Reduction in physical fitness. Increase in perception } \\
\text { of work as physically strenuous }\end{array}$ & $76 \%$ \\
\hline Liungberg et al $(1989)^{28}$ Sweden & $\begin{array}{l}2,6,7 \\
\text { Nursing staff }(n=24)\end{array}$ & $\begin{array}{l}\text { Liffing rates, cumulative force; total liffing time, and no. } \\
\text { of steps while carrying }\end{array}$ & $\begin{array}{l}\text { Modern ward showed a reduction in: lifting rates }(50 \%) \text {; cumulative force } \\
(57 \%) \text {; total lifting time }(78 \%) \text {; no. of steps while carrying }(72 \%)\end{array}$ & $65 \%$ \\
\hline Lynch and Freund $(2000)^{62}$ USA & $\begin{array}{l}5 \\
\text { Nursing staff }(n=374)\end{array}$ & $\begin{array}{l}\text { Knowledge about back injury risk factors } \\
\text { Change in work practices } \\
\text { Lost time back iniuries }\end{array}$ & $\begin{array}{l}\text { No change in level of knowledge } \\
\text { Repositioning in-bed tasks reduced } \\
30 \% \text { reduction in lost time back iniuries over previous } 3 \text { years }\end{array}$ & $50 \%$ \\
\hline Menckel et al $(1997)^{19}$ Sweden & $\begin{array}{l}1,2,5,8 \\
\text { Health care staff }(n=122)\end{array}$ & Implementation of feedback & $42 \%$ of measures were implemented & $63 \%$ \\
\hline Miller and Johnson $(1992)^{20}$ UK & $\begin{array}{l}1,5,10 \\
\text { Home care staff }(n=10)\end{array}$ & Questionnaire & $\begin{array}{l}\text { Increase in qualitative measures of carer confidence and feeling of control } \\
\text { of situation }\end{array}$ & $50 \%$ \\
\hline Monoghan et al $(1998)^{25} \mathrm{UK}$ & $\begin{array}{l}1,2,5,10,13 \\
\text { Nurses }(n=28)\end{array}$ & $\begin{array}{l}\text { Training attendance } \\
\text { Patient assessment plans }\end{array}$ & $\begin{array}{l}59 \% \text { attendance } \\
75 \% \text { of patients had mobility plans }\end{array}$ & $31 \%$ \\
\hline Nussbaum and Torres $(2001)^{50}$ USA & $\begin{array}{l}5 \\
\text { Nurses }(n=24)\end{array}$ & $\begin{array}{l}\text { RPE } \\
\text { Postural analysis } \\
\text { Biomechanical analysis }\end{array}$ & No significant change & $59 \%$ \\
\hline Nyran (1991) $)^{21}$ Canada & $\begin{array}{l}1,2,4,5 \\
\text { Nursing Homes }(\mathrm{n}=48)\end{array}$ & $\begin{array}{l}\text { Cost effectiveness } \\
\text { Lost time claims (Compensation Board) }\end{array}$ & Net saving of $\$ 57,439$ & $65 \%$ \\
\hline Oddy $(1993)^{29}$ UK & $\begin{array}{l}3,6,10,13 \\
\text { Continuing care ward }(n=24)\end{array}$ & Elimination of drag lift & Reduction over 6 months, with alternative techniques used & $50 \%$ \\
\hline
\end{tabular}




\begin{tabular}{|c|c|c|c|c|}
\hline Author & Intervention subjects $(\mathrm{n})$ & Outcome measures & Results & QR \\
\hline Paternoster et al $(1999)^{73}$ Italy & $\begin{array}{l}5,18 \\
\text { Hospital workers }(n=80)\end{array}$ & Postural analysis & Incorrect postures reduced from $68 \%$ to $38 \%$ & $31 \%$ \\
\hline Peers $(1998)^{26}$ Canada & $\begin{array}{l}5,10,13,15,20 \\
\text { Nursing home staff }(n=131)\end{array}$ & Lost time and modified work duties & $\begin{array}{l}\text { Lost time reduced from } 249 \text { to } 30 \text { days } \\
\text { Modified work days reduced from } 246 \text { to } 184\end{array}$ & $37 \%$ \\
\hline Pohjonen et al $(1998)^{22}$ Finland & $\begin{array}{l}1,2,3,7,9,10,11 \\
\text { Home care staff }(n=70)\end{array}$ & $\begin{array}{l}\text { Postural analysis } \\
\text { Heart rate } \\
\text { Psychosocial questionnaire (Work Ability Index) }\end{array}$ & $\begin{array}{l}\text { Significant increase in proportion of straight back positions (from } 59 \text { to } \\
75 \% \text { ) } \\
\text { No change in heart rate data or psychosocial data for intervention group }\end{array}$ & $58 \%$ \\
\hline Rodgers $(1985)^{59}$ UK & $\begin{array}{l}5 \\
\text { Ward staff ( } n=4 \text { wards) }\end{array}$ & Use of taught lifting techniques & $\begin{array}{l}\text { Shoulder lift not used } \\
30 \% \text { of } 2 \text {-person lifts carried out by one person }\end{array}$ & $38 \%$ \\
\hline Santoro $(1994)^{43}$ USA & $\begin{array}{l}17 \\
\text { Neurology staff }(n=65)\end{array}$ & Effectiveness of lifting team & $90 \%$ of lifts achieved & $35 \%$ \\
\hline Scholey $(1983)^{70}$ UK & $\begin{array}{l}5 \\
\text { Nurses }(n=4)\end{array}$ & Intra abdominal pressure (IAP) & Significant reduction in IAP & $78 \%$ \\
\hline Scopa $(1993)^{51}$ USA & $\begin{array}{l}5 \\
\text { Nurses }(n=49)\end{array}$ & Evaluation of body mechanics & No significant difference & $65 \%$ \\
\hline Stubbs et al $(1983)^{52}$ UK & $\begin{array}{l}5 \\
\text { Student nurses }(n=2)\end{array}$ & Intra abdominal pressure & Minimal reduction in IAP at best, deterioration at worst & $55 \%$ \\
\hline St Vincent et al $(1989)^{53} \mathrm{UK}$ & $\begin{array}{l}5 \\
\text { Orderlies }(n=33)\end{array}$ & Use of taught handling methods (6 principles) & $\begin{array}{l}\text { Application of all } 6 \text { principles only in } 1 \% \text { of sample. Frequency of use of } \\
\text { individual principles ranged between } 11-33 \%\end{array}$ & $70 \%$ \\
\hline Torri et al (1999) ${ }^{30}$ Italy & $\begin{array}{l}2,5 \\
\text { Hospital staff ( } n=\text { approx. } 900 \text { ) }\end{array}$ & $\begin{array}{l}\text { Sickness absence } \\
\text { Use of hoists (lifters) }\end{array}$ & $\begin{array}{l}\text { Reduction in sickness absence (39\%) } \\
71 \% \text { used hoists regularly and correctly }\end{array}$ & $50 \%$ \\
\hline Tracz and Rose $(1982)^{35}$ Canada & $\begin{array}{l}2,5 \\
\text { Rehabilitation ward staff ( } n=?)\end{array}$ & $\begin{array}{l}\text { Reported injuries } \\
\text { Lost time for back injuries }\end{array}$ & Little change & $33 \%$ \\
\hline Trevelyan $(2001)^{36} \mathrm{UK}$ & $\begin{array}{l}2,5,7,10 \\
\text { Nurses }(n=48)\end{array}$ & $\begin{array}{l}\text { Self-reported well-being questionnaire } \\
\text { Task and postural analysis }\end{array}$ & No significant difference for any of the measures & $78 \%$ \\
\hline Troup and Rauhala $1987^{54}$ UK and Finland & $\begin{array}{l}5 \\
\text { Student nurses ( } n=4 \text { groups) }\end{array}$ & $\begin{array}{l}\text { Use of taught techniques } \\
\text { Back injuries }\end{array}$ & $\begin{array}{l}\text { New skills were acquired and increased use of equipment } \\
\text { No significant difference in prevalence or incidence of back pain and } \\
\text { injuries }\end{array}$ & $54 \%$ \\
\hline Tuffnell $(1989)^{74}$ New Zealand & $\begin{array}{l}5,10 \\
\text { Nurses }(n=?)\end{array}$ & Type of lifts & Increase in use of shoulder lift from 6 to $50 \%$ & $30 \%$ \\
\hline Videman et al $(1989)^{60}$ Finland & $\begin{array}{l}5 \\
\text { Student nurses }(n=200)\end{array}$ & $\begin{array}{l}\text { Skill assessment } \\
\text { Prevalence and incidence of back pain and injuries }\end{array}$ & $\begin{array}{l}\text { Improvement in skills for techniques (63\%) and lifting aids (53\%) used } \\
\text { No significant difference in prevalence or incidence of back pain and } \\
\text { injuries }\end{array}$ & $41 \%$ \\
\hline Wachs and Parker $(1987)^{47}$ USA & $\begin{array}{l}5 \\
\text { Nursing staff }(n=178)\end{array}$ & $\begin{array}{l}13 \text { point skills checklist (environmental factors and } \\
\text { postural assessment) }\end{array}$ & $\begin{array}{l}\text { Low level of prescribed lifting behaviours }(17 \%) \text {, only } 2 \% \text { completed all } 13 \\
\text { prescribed behaviours. } 23 \% \text { of postures were labelled 'at risk' }\end{array}$ & $86 \%$ \\
\hline Wood et al $(2000)^{75}$ USA & $\begin{array}{l}5 \\
\text { Nursing assistants }(n=90)\end{array}$ & $\begin{array}{l}\text { Evaluation of transfer skills } \\
\text { Audit of bedside information }\end{array}$ & $\begin{array}{l}\text { Prescribed techniques were performed } 68 \% \text { of the time } \\
37 \% \text { of bedside information was accurate }\end{array}$ & $46 \%$ \\
\hline Wood $(1987)^{55}$ Canada & $\begin{array}{l}5,8 \\
\text { Nursing staff ( } n=3 \text { units) }\end{array}$ & $\begin{array}{l}\text { No. of wage loss claims for back injuries caused by } \\
\text { interactions with residents }\end{array}$ & No significant difference between expt. and control groups (both reduced) & $56 \%$ \\
\hline \multicolumn{2}{|c|}{$\begin{array}{l}\text { Key } \\
\text { Intervention strategy included: } \\
1=\text { Risk assessment } \\
2=\text { Equipment provision or/and purchase (including training in new equipment) } \\
3=\text { Equipment design/evaluation } \\
4=\text { Equipment maintenance } \\
5=\text { Education and training } \\
6=\text { Work environment redesign, space constraints addressed } \\
7=\text { Work organisation/practices changed } \\
8=\text { Feedback } \\
9=\text { Group problem solving/team building } \\
10=\text { Review and change of policies and procedures/safe systems of work } \\
11=\text { Discussion of goals with clients }\end{array}$} & \multicolumn{2}{|c|}{$\begin{array}{l}12=\text { Injury monitoring system with follow up. Return to work programme } \\
13=\text { Change/introduction of patient assessment system } \\
14=\text { Introduction of hazard register } \\
15=\text { Audit of working practices/risk assessments } \\
16=\text { Review of staffing levels. Increase in staffing level } \\
17=\text { Introduction of lifting team programme } \\
18=\text { Physical fitness training } \\
19=\text { Stress management } \\
20=\text { Medical examination and lifting skill assessment } \\
21=\text { Task analysis, job design analysis } \\
22=\text { Change in uniforms }\end{array}$} & \\
\hline
\end{tabular}

= Injury monitoring system with follow up. Return to work programme

$4=$ Introduction of hazard register

$6=$ Review of staffinges/risk assessmentis

$17=$ Introduction of lifting team programme

$9=$ Stress management

$21=$ Task analysis, job design analysis 
Table 3 Most commonly used strategies in multifactor interventions

\begin{tabular}{lll}
\hline Intervention strategy (key reference number) & No. of occurrences & Average QR of studies \\
\hline Equipment provision/purchase (2) & 18 & $50 \%$ \\
Education and training (e.g. risk assessment, use of & 18 & $54 \%$ \\
equipment, patient assessment) (5) & 13 & $55 \%$ \\
Risk assessment (1) & 10 & $50 \%$ \\
Policies and procedures (10) & 8 & $43 \%$ \\
Patient assessment system (13) & 7 & $58 \%$ \\
Work environment redesign (6) & 7 & $63 \%$ \\
Work organisation/practices changed (7) &
\end{tabular}

results is supported with moderate evidence from two studies. ${ }^{61}{ }^{62}$ Additional support is given at the limited level from four studies. ${ }^{63-66}$

++ The evidence statement that interventions based on technique training can have short term positive outcomes is supported with moderate evidence from four studies.$^{67-70}$ Limited evidence is available from another five studies. ${ }^{71-75}$ However, all these studies reported either procedural difficulties with a lack of control groups, use of different workers and/or patients pre/post intervention, or that statistical significance was not achieved.

\section{DISCUSSION}

International evidence was found for a range of intervention strategies. The results have been summarised as evidence statements to group the papers into three categories: multifactor interventions, single factor interventions, and interventions based on technique training.

\section{Multifactor interventions}

The multifactor intervention strategies included risk assessment, equipment provision, equipment evaluation/design, equipment maintenance, education and training, work environment redesign, work organisation/practices changed, feedback, group problem solving/team building, review and change of policies and procedures, discussion of goals with clients, injury monitoring systems (return to work programmes), patient assessment systems, hazard registers, audit of working practices/risk assessments, physical fitness training, and medical examinations.

The papers in this category were subgrouped to look at whether they included a risk assessment programme which, although not an intervention in itself, has an important role to play as an integral part of an intervention. The evidence statement for interventions, including a risk assessment is supported by 14 studies at the moderate and limited levels. The risk assessment programme could include feedback to staff and supervisors and the discussion of goals with clients. Some also gave evidence of audit of either working practices and/or the risk assessment programme. It is suggested that risk assessment (in the context of interventions to reduce risks associated with patient handling) provides the framework which is needed for an intervention to be embedded within an organisation's structure and culture. ${ }^{76} 77$

The second subgroup (no risk assessment) includes 10 studies, with an overall lower level of evidence (only four studies at the moderate level) and one contradictory high quality study. ${ }^{36}$ These interventions were generally preplanned or expert led. Both subgroups included programmes as short as 6 months and as long as 3-5 years, so the duration of the intervention is unlikely to contribute to the different findings. The conclusion for this category is that although multifactor interventions may show some improvements, they are more likely to succeed if they are based on a risk assessment programme (involving the staff).

\section{Single factor interventions}

The single factor interventions are divided into the provision of equipment (moderate evidence from only two studies) and the lifting team approach. Although it is unusual to find only equipment provision without other factors, if the provision of hoisting equipment can be shown, in future high quality research, to have a significant impact on robust outcome measures (for example, local measures of physiological changes as well as organisational measures looking at sickness absence and incident reports), single factor interventions based on equipment provision might prove to be more cost effective than multifactor interventions.

The second single factor intervention is the lifting team approach which has an evidence statement supported at the moderate level. Currently the research for this approach is only available from the USA, so it might be interesting to see if the results can be replicated in other countries.

\section{Technique training based interventions}

Finally the third category, interventions predominantly based on technique training, has also been divided into three subgroups. The strongest support is for the evidence statement that interventions predominantly based on technique training have no impact on working practices or injury rates. This is supported with the highest level of evidence (strong) from four studies with an additional 13 studies at the moderate and limited levels. However, evidence was also found supporting the opposing statement for the use of training, but only to achieve short term changes, with four studies at a moderate level and five studies at the limited level.

\section{Generic multifactor intervention programme}

The 22 multifactor interventions from categories (1) and (2) included 19 strategies, in different combinations. These have been further analysed as shown in table 3, listing the seven most commonly used. The average QR score is given for each intervention strategy. Studies using work organisation/ practice change have the highest average score (63\%) and those incorporating a patient assessment system, the lowest $(43 \%)$.

It is suggested that these top seven factors could form the basis of a generic programme, although it is likely that an intervention strategy and programme will need to be further developed and extended in order to be responsive to local organisational and cultural factors. The risk assessment process could facilitate the detailed design of the programme, and identification of additional appropriate strategies, with the allocation of priorities based on local negotiation with managers and staff.

\section{Cost effectiveness}

The cost effectiveness of interventions was only reported for two studies, ${ }^{21}{ }^{42}$ with \$55 000-65000 annual savings. These used a multifactor intervention programme, including risk assessment ${ }^{21}$ and the lifting team ${ }^{42}$ strategy. 


\section{Conclusion}

This systematic review has drawn together international data relating to patient handling interventions from 1960 to 2001. There is strong evidence against interventions predominantly based on technique training. It is suggested that the seven most commonly used strategies from the multifactor interventions could form the basis of a generic programme, with additional strategies being identified through the risk assessment process. However, the programmes using single factor interventions (hoisting equipment and lifting teams) also provided a moderate level of evidence and it may be, with more high quality research, that these may be shown to offer more cost effective strategies. Unfortunately, as only two studies from the USA reported data on financial savings, it will be difficult for health care managers to draw conclusions from these data as the financial accounting systems (for example, workers' compensation and insurance) may be different.

The main recommendation from these findings is that health care providers should review their current approach to managing risks and injuries associated with patient handling activities. If their approach is predominantly based on technique training it is unlikely to be successful in reducing musculoskeletal injuries, and an alternative intervention strategy should be considered.

\section{ACKNOWLEDGEMENTS}

Research funding: Financial support for this project was received from the Health and Safety Executive (RSU ref. 4160/R55.092) and the NHS Executive (Trent) (ref. RBG 01XX3).

The valuable contributions of the following are gratefully acknowledged: Dr Michael Dewey, Deputy Director of Trent Institute for Health Services Research, University of Nottingham; Dr Julie Glanville, Associate Director, NHS Centre for Reviews and Dissemination of Information, University of York; Dr John Rule, Library Manager, Nottingham City Hospital NHS Trust.

Contributors: Data were collected using the extraction/appraisal tool by the project team: Sue Hignett, Emma Crumpton, Sue Ruszala, Pat Alexander, Mike Fray, and Brian Fletcher.

\section{REFERENCES}

1 Smedley J, Egger P, Cooper C, et al. Manual handling activities and risk of low back pain in nurses. Occup Environ Med 1995:52:160-3.

2 Hignett S. Work-related back pain in nurses. J Adv Nurs 1996; 23: 1238-46

3 Lloyd P, Fletcher, Holmes, et al. The Guide to the Handling of Patients, 4th edn. National Back Pain Association/Royal College of Nursing, 1998.

4 Disabled Living Foundation. Handling people: equipment, advice and information, 2nd edn. London: Disabled Living Foundation, 2001

5 Chartered Society of Physiotherapy. Moving and handling for chartered physiotherapists. London: Chartered Society of Physiotherapy, 1998.

6 Royal College of Midwives. Handle with care. A midwife's guide to preventing back injury. London: Royal College of Midwives, 1997.

7 The Resuscitation Council. Guidance for safer handling during resuscitation in hospital. London: The Resuscitation Council, 2001.

8 Faculty of Occupational Medicine. Occupational health guidelines for the management of low back pain at work. Evidence review and recommendations. London: Faculty of Occupational Medicine, 2000:27-8.

9 Hignett S, Crumpton E, Ruszala S, et al. Evidence based patient handling: tasks, equipment and interventions. London: Routledge, 2003.

10 Downs SH, Black N. The feasibility of creating a checklist for the assessment of the methodological quality both of randomised and non-randomised studies of health care interventions. J Epidemiol Community Health 1998;52:377-84.

11 Crumpton E, Hignett S, Goodwin R, et al. Establishing reliability for data extraction in a systematic review on patient handling. In: McCabe PT, ed. Contemporary ergonomics. London: Taylor and Francis, 2002:51-6.

12 Bernard BP, ed. Musculoskeletal disorders and work place factors. A critical review of epidemiologic for work-related musculoskeletal disorders of the neck, upper extremities and low back. NIOSH: US Department of Health and Human Sciences, 1997:1.13-1.14.

13 Alexander P. Risk management in manual handling for community nurses. In: Hanson M, ed. Contemporary ergonomics. London: Taylor and Francis, 1998:87-91.
14 Collins M. A comprehensive approach to preventing occupational back pain among nurses. Journal of Occupational Health \& Safety-Australia \& New Zealand 1990;6:361-8

15 Duggan EA. An ergonomics approach to the reduction of the physical load of some nurses. Unpublished MSc dissertation, University of Limerick, 1995.

16 Evanoff BA, Bohr PC, Wolf LD. Effects of a participatory ergonomics team among hospital orderlies. Am J Ind Med 1999;35:358-65.

17 Garg A, Owen B. Reducing back stress in nursing personnel: an ergonomic intervention in a nursing home. Ergonomics 1992:35:1353-75.

18 Hignett S, Richardson B. Manual handling human loads in a hospital: an exploratory study to identify nurses' perceptions. Appl Ergon 1995;26:221-6.

19 Menckel E, Hagberg M, Engkvist IL, et al. The prevention of back injuries in Swedish health care-a comparison between two models for action-oriented feedback. Appl Ergon 1997;28:1-7.

20 Miller $\mathbf{M}$, Johnston C. Moving and handling skills for carers. TES Crossroads, 1992:1-28.

21 Nyran PI. Cost effectiveness of core-group training. In: Karwowski W, Yates JW, eds. Advances in industrial ergonomics and safety III. Taylor \& Francis, 1991:778-82.

22 Pohjonen T, Punakallio A, Louhevaara V. Participatory ergonomics for reducing load and strain in home care work. Int $J$ Ind Ergon 1998;21:345-52.

23 Goodridge D, Laurila B. Minimizing transfer injuries. The Canadian Nurse 1997;93(7):38-41

24 Head M, Levick P. Patient handling: an ergonomic intervention. In: Proceedings of the 32nd Annual Ergonomics Society of Australia and the Safety Institute of Australia National Conference, "Enhancing Human Performance", 22-25 September 1996, Canberra, Australia.

25 Monaghan $\mathbf{H}$, Robinson L, Steele Y. Implementing a no lift policy. Nursing Standard 1998;12(50):35-7.

26 Peers ML. Prevention of nursing strain injuries in the long term care setting: the Fairview Lodge experience. Perspectives 1998;22(1):23-4

27 Daynard D, Yassi A, Cooper JE, et al. Biomechanical analysis of peak and cumulative spinal loads during simulated patient-handling activities: a sub-study of a randomised controlled trial to prevent lift and transfer injury of health care workers. Appl Ergon 2001;32:199-214.

28 Ljungberg AS, Kilborn A, Hägg G. Occupational lifting by nursing aides and warehouse workers. Ergonomics 1989;32(1):59-78.

29 Oddy, R. Legislation in action: must we lift? Physiotherapy 1993;79:827-30

30 Torri $\mathbf{P}$, Liboni $\mathrm{D}$, Milan $\mathrm{F}$, et al. An experience in management of risk due to manual lifting of patients in hospitals in the Veneto Region [in Italian]. Medicina del Lavoro 1999:90:362-80.

31 Aird JW. Comprehensive back injury prevention programme: an ergonomic approach for controlling back injuries in health care facilities In: Aghazadeh F, ed. Trends in ergonomics human factors $V$. Holland: Elsevier Science, 1988:705-12.

32 Dixon R, Lloyd B, Coleman S. Defining and implementing a no lifting standard. Nursing Standard 1996;10(44):33-6.

33 Entwhistle $M$, Horman $M$, Lister L, et al. Safe manual lifting of patients. J Assoc Qual Healthcare 1996;3(3): 1 18-24.

34 Kilbom A, Ljungberg AS, Hägg G. Lifting and carrying in geriatric care. A comparison between differences in workspace layout, work organisation and use of modern equipment. In: Brown I, et al, eds. Ergonomics International 1985:85:550-3.

35 Tracz S, Rose I. Beating low back pain. Dimensions in Health Service 1982;59:20-3

36 Trevellyan FC. The implementation and evaluation of an ergonomics intervention in a health care setting. Unpublished PhD thesis. Robens Centre for Health Ergonomics, European Institute for Health and Medical Sciences, University of Surrey, 2001.

37 Holliday PJ, Fernie GR, Plowman S. The impact of new lifting technology in long term care. AAOHN Journal 1994;42:582-9.

38 Knibbe JJ, Friele RD. The use of logs to assess exposure to manual handling of patients, illustrated in an intervention study in home care nursing. Int $J$ Ind Ergon 1999;24:445-54.

39 Caska BA, Patnode RE, Clickner A. Feasibility of a nurse staffed lift team. D. AAOHN 1998:46:283-8.

40 Charney W. The lift team method for reducing back injuries: A 10 hospital study. AAOHN Journal 1997;45:300-4

41 Charney W, Zimmerman K, Walara E. A design method to reduce lost time back injury in nursing. Chapter 8 . In: Charney W, Schirmer J, eds. Essentials of modern hospital safety, Volume 2. Lewis Publishers, 1993:313-24

42 Charney W, Zimmerman K, Walara E. The lifting team. A design method to reduce lost time back injury in nursing. AAOHN Journa 1991;39:231-4

43 Santoro $M$. Lifting teams can help hospitals eliminate costly back injuries to nurses. Hospital Employee Health 1994;13:81-7.

44 Engkvist I-L, Kjellberg A, Wigaeus HE, et al. Back injuries among nursing personnel-identification of work conditions with cluster analysis. Safety Science 2001;37:1-18.

45 Fanello S, Frampas-Chotard V, Roquelaure $Y$, et al. Evaluation of an educational low back pain prevention program for hospital employees. Revue Du Rhumatisme, English Edition 1999;66:711-16.

46 Lagerström M, Hagberg M. Evaluation of a three year education and training program for nursing personnel at a Swedish Hospital. AAOHN 1997;45(2):83-92

47 Wachs JE, Parker JE. Registered nurses' lifting behaviour in the hospital setting. In: Asfour S, ed. Trends in ergonomics/human factors IV. North Holland: Elsevier Science Publishers BV, 1987:883-90. 
48 Billin SL. Moving and handing practice in neuro-disability nursing. $\mathrm{Br}$ J Nurs 1998;7:571-8

49 Harber P, Pena L, Hsu P, et al. Personal history, training, and worksite as predictors of back pain of nurses. Am J Ind Med 1994;25:519-26.

50 Nussbaum MA, Torres $\mathrm{N}$. Effects of training in modifying working methods during common patient-handling activities. Int J Ind Ergon 2001;27:33-41.

51 Scopa M. Comparison of classroom instruction and independent study in body mechanics. The Journal of Continuing Education in Nursing 1993:24:170-3.

52 Stubbs DA, Buckle P, Hudson MP, et al. Back pain in the nursing profession-II. The effectiveness of training. Ergonomics 1983;26:767-9.

53 St Vincent $M$, Tellier $C$, Lortie $M$. Training in handling an evaluative study. Ergonomics 1989;32:191-210.

54 Troup JD, Rauhala HH. Ergonomics and training. International Journal of Nursing Studies 1987;24:325-30.

55 Wood DJ. Design and evaluation of a back injury prevention program within a geriatric hospital. Spine 1987;12:77-82.

56 Dietz E, Baumann M. Obstacles to change: discussion and points of view of health care professionals with regard to the application patient-handling training. Archives des Maladies Professionnelles et de medecine du Travail 2000;61:389-95

57 Griffith J, McArthur M. A qualitative study exploring the meaning of manual handing training in a working environment at risk of back injury. Advancing Clinical Nursing 1999;3:179-86.

58 Johnston M. Handle with care. Senior Nurse 1987;6(5):20-2.

59 Rodgers S. Positive lifting. Nursing Times 23 January 1985:43-5.

60 Videman T, Rauhala H, Asp S, et al. Patient-handling skill, back injuries, and back pain: an intervention study in nursing. Occup Health 1989;41:148-56.

61 Hellsing AL, Linson SJ, Andershed B, et al. Ergonomic education for nursing students. International Journal of Nursing Studies 1993;30:499-510.

62 Lynch RM, Freund A. Short-term efficacy of back injury intervention project for patient care providers at one hospital. AlHAJ. Journal for the Science of Occupational \& Environmental Health \& Safety 2000;61:290-4
63 Addington C. All the right moves. A program to reduce back injuries in OR nurses. AORN J 1994;59:483-8.

64 Daws J. Lifting and moving patients. 3. A revision training programme. Nursing Times 25 November 1981:2067-9.

65 Engels JA, van der Gulden JW, Senden TF, et al. The effects of an ergonomic-educational course. Postural load, perceived physical exertion, and biomechanical errors in nursing. Int Arch Occup Environ Health 1998;71:336-42.

66 Garrett RB, Johnson Perry A. A safer way to move patients. Occupational Health and Safety 1996:60-4.

67 Best $M$. An evaluation of manutention training in preventing back strain and resultant injuries in nurses. Safety Science 1997;25:207-22.

68 Feldstein A, Valanis B, Vollmer W, et al. The Back Injury Prevention Project pilot study. Assessing the effectiveness of back attack, an injury prevention program among nurses, aides, and orderlies. J Occup Med 1993:35:114-20

69 Foster L. Manual handing training and changes in work practices. Occup Health 1966;48:402-6.

70 Scholey $M$. Back stress; the effects of training nurses to lift patients in a clinical situation. International Journal of Nursing Studies 1983:20:1-13.

71 Alavosius MP, Sulzer-Azaroff B. The effects of performance feedback on the safety of client lifting and transfer. J Appl Behav Anal 1986;19:261-7.

72 Gray J, Cass J, Harper DW et al. A controlled evaluation of a lifts and transfer educational program for nurses Geriatric Nursing 1996;17(2):81-5.

73 Paternoster D, Salis M, Gisser GV. An experience in training of hospital staff with tasks involving manual load lifting in Bressanone Hospital: content and checking of efficacy [in Italian]. Medicina del Lavoro 1999:90:381-92.

74 Tuffnell C. Lightening the lifting load through education and quality assurance monitoring. Aust Clin Rev 1989;9:123-6.

75 Wood J, Raudsepp T, Miller L, et al. Improving resident transfers. Nursing Homes Long Term Care Management 2000:68-76.

76 Hignett S. Embedding ergonomics in hospital culture: top-down and bottom-up strategies. Appl Ergon 2001;32:61-9.

77 Helmreich RL, Merritt AC. Culture at work in Aviation and Medicine. Aldershot: Ashgate Publishing Ltd, 1998. 